\title{
Characteristics of cathelicidin-Bg, a novel gene expressed in the ear-side gland of Bufo gargarizans
}

\author{
F. Gao ${ }^{1,2}$, W.F. Xu ${ }^{1}$, L.P. Tang ${ }^{1}$, M.M. Wang ${ }^{1}$, X.J. Wang ${ }^{1,2}$ and Y.C. Qian ${ }^{1,3}$ \\ ${ }^{1}$ Traditional Chinese Medicine Unit, School of Forestry and Biotechnology, \\ Zhejiang A\&F University, Lin'An, Zhejiang, China \\ ${ }^{2}$ Nurturing Station for the State Key Laboratory of Subtropical Silviculture, \\ Zhejiang Agricultural and Forestry University, Lin'an, China \\ ${ }^{3}$ Department of Veterinary Integrative Biosciences, Texas A\&M University, \\ TX, USA
}

Correspondent authors: Y.C. Qian / X.J. Wang

E-mail: qian3906@zafu.edu.cn / gfei1981@live.com

Genet. Mol. Res. 15 (3): gmr.15038481

Received January 25, 2016

Accepted March 11, 2016

Published August 5, 2016

DOI http://dx.doi.org/10.4238/gmr.15038481

Copyright (C) 2016 The Authors. This is an open-access article distributed under the terms of the Creative Commons Attribution ShareAlike (CC BY-SA) 4.0 License.

ABSTRACT. The traditional Chinese medicine Chan Su (toad venom)
comprises dried secretions of the ear-side gland of Bufo gargarizans.
Chan Su is known for its small molecular components, which include
telocinobufagin, marinobufagin, and bufalin, while in other amphibians,
studies mainly focus on peptide components. Until recently, no genes
expressed in the ear-side gland of B. gargarizans gland had been cloned.
In this study, cathelicidin-Bg, a coding sequence of anti-microbial
peptide (AMP), was cloned. The predicted amino acid sequence of
cathelicidin-Bg was very similar to that from other amphibians, with
a $34-$ amino acid mature peptide predicted in the C-terminus. The
functions of this mature peptide were verified by microbe and tumor 
cell inhibition assays. Our results showed that the mature peptide of cathelicidin-Bg could inhibit the proliferation of Staphylococcus aureus and Pseudomonas aeruginosa. The mature peptide was also shown to selectively inhibit tumor cells. These results indicate that the identified coding sequence represents an active peptide of Chan $\mathrm{Su}$.

Key words: Cathelicidin-Bg; Bufo gargarizans; Anti-microbial peptide; Tumor

\section{INTRODUCTION}

Peptides secreted from amphibian skin have been the focus of many studies (Miller, 1990; Simmaco et al., 1990; Lee et al., 2005; King et al., 2008). In recent years, a large number of anti-tumor peptides have been reported in different toad and frog species (Bevins and Zasloff, 1990; Bechinger et al., 1993; Westerhoff et al., 1995). Members of the maganin family of peptides have been isolated from the skin of African clawed frog Xenopus laevis (Ohsaki et al., 1992; Baker et al., 1993). In addition, the 23-amino acid peptides maganin 1 and 2 have been reported to inhibit proliferation of NCI-H82 and NCI-H526 cells at very low doses (Baker et al., 1993; Giacometti et al., 2004). Because maganin 2 is highly cytotoxic, its derivative, formed via amino acid modification, has been approved as a novel cancer treatment (Shin et al., 2000; Takeshima et al., 2003). Furthermore, peptides belonging to the maximin and bombinin families in Bombina maxima and Bombina orientalis have been reported to inhibit the proliferation of tumor cells (Gibson et al., 1991).

In contrast to the anti-tumor activity of peptides from other amphibians, studies on the anti-tumor activity of skin secretion from Bufo gargarizans have focused on small molecules. In traditional Chinese medicine, dried secretions from the skin gland of $B$. gargarizans (Chan $\mathrm{Su}$ ) have been used as an anti-inflammatory agent in China for thousands of years (The State Pharmacopoeia Commission of PR China, 2010). Recent studies have reported that toad venom has anti-tumor activity. For example, toad venom extracts were shown to inhibit the growth of non-small-cell lung cancer (Meng et al., 2009; Zhang et al., 2013b). Active components of Chan Su such as telocinobufagin, marinobufagin, bufalin, bufotalin, and resibufogenin were reported to have anti-tumor activity (Qiao et al., 2008; Li et al., 2010; Zhang et al., 2013a). Bufalin and cardiotonic steroids isolated from $\mathrm{Chan} \mathrm{Su}$ were found to promote apoptosis in human prostate and breast cancer cells by up-regulating the expression of caspase family genes (Zhu et al., 2012; Hong et al., 2013). Bufalin can also suppress cellular proliferation and inhibit the migration and invasion of tumor cells, although the molecular mechanisms underlying these effects remain unknown (Zhai et al., 2013; Chang et al., 2014; Chen et al., 2015). Furthermore, arenobufagin, another major component of $\mathrm{Chan} \mathrm{Su}$, was reported to be a specific inhibitor of vascular endothelial growth factor-mediated angiogenesis (Zhang et al., 2013a).

Although no coding sequence has been cloned from the ear-side gland, which secretes $\mathrm{Chan} \mathrm{Su}$, the coding sequence of anti-microbial peptide (AMP) has been identified from the skin of B. gargarizans, e.g., cathelicidin (Sun et al., 2015), which is a homolog identified in other amphibians. In this study, we constructed a cDNA library using total RNA isolated from the ear-side gland of $B$. gargarizans, and attempted to use it to identify the coding sequences of AMPs in order to identify novel components of Chan $\mathrm{Su}$.

Genetics and Molecular Research 15 (3): gmr.15038481 


\section{MATERIAL AND METHODS}

\section{Toad samples}

B. gargarizans weighing 100-130 g were collected from Hangzhou, China on the first day of each month from April to November in 2015. Toads were surface-sterilized using $75 \%$ ethanol and ear-side glands were subsequently dissected. Total RNA was extracted from the ear-side glands using the TRIzol (Invitrogen ${ }^{\mathrm{TM}}$, USA) method. Then, first-strand cDNA was synthesized by ReverTra Ace qPCR RT Kit (TOYOBO, Japan). For gene cloning, a mixed sample of samples from each month was used and individual samples were used to analyze gene expression profiles.

\section{Sequencing of a partial DNA sequence}

To obtain a partial cDNA sequence, primers (Table 1) were designed based on cathelicidin sequences conserved in other amphibian species. Synthesized cDNA was used as a template, and PCR was conducted in the following manner: $94^{\circ} \mathrm{C}$ for $5 \mathrm{~min}$, followed by 35 cycles at $94^{\circ} \mathrm{C}$ for $30 \mathrm{~s}, 68^{\circ} \mathrm{C}$ for $30 \mathrm{~s}, 72^{\circ} \mathrm{C}$ for $1 \mathrm{~min}$, and $72^{\circ} \mathrm{C}$ for $7 \mathrm{~min}$. A generated DNA fragment of the expected size was used to construct a recombinant plasmid with a pMD-19 T vector (TaKaRa, Japan), which was transferred to Escherichia coli DH5 $\alpha$ cells, and sequenced by Sangon Biotech Co. Ltd. (Shanghai, China).

\section{Table 1. Primers used for gene cloning and differential expression of Cathelicidin-Bg.}

\begin{tabular}{l|l|c|c}
\hline Primers & Primer sequences & Application & Accession No. \\
\hline RACE-Cat-5' & 3'-CTTGGACCTCAGGCTCTGCAGT-5' & RACE & KU556733 \\
\hline RACE-Cat-3' & 3'-GGATCCTGAGGATATAATGTGCACC-5' & RACE & KU556733 \\
\hline q-Cat-F & 3'-ATTAAAGAGACGGTGTGCCTCAAAT-5' & qPCR & KU556732 \\
\hline q-Cat-R & 3'-TGTTGGACCGCTTCACACGAAC-5' & qPCR & KU556732 \\
\hline q-actin-F & 3'-GAGCTATGAGTTGCCTGATGGACAG-5' & qPCR & EU661596 \\
\hline q-actin-R & 3'-AATCCTTACGAATATCCACATCACAC-5' & qPCR & EU661596 \\
\hline
\end{tabular}

\section{Rapid amplification of cDNA end (RACE)}

The full-length cathelicidin-Bg cDNA sequence was cloned by the RACE method. Primers (Table 1) were designed from partial cathelicidin-Bg sequences (accession No. KU556733), as described above. Synthesized cDNA was used as a template, and 3'- and 5'RACE was performed using a SMART ${ }^{\mathrm{TM}}$ RACE cDNA Amplification Kit (Clontech, USA) according to the manufacturer instructions. PCR fragments were subcloned into the pMD-19 $\mathrm{T}$ vector, transferred to $E$. coli $\mathrm{DH} 5 \alpha$ cells, and sequenced by Sangon Biotech.

\section{Analysis of cDNA sequence}

BLASTn and BLASTp were used to identify the nucleotide and deduced amino acid sequences of the cloned cDNA sequence. A putative signal peptide was predicted by the SignalP 4.1 Server online software. Multiple alignments between cathelicidin-Bg and its homologs were conducted using the DNAMAN software, and nucleotide and deduced amino acid sequence profiles were analyzed by the DNASTAR software. A phylogenetic tree was constructed using the neighbor-joining algorithm in the MEGA software.

Genetics and Molecular Research 15 (3): gmr.15038481 
F. Gao et al.

\section{Quantitative PCR (qPCR)}

To normalize differences in the amount of reverse-transcribed RNA used in each reaction, $\beta$-actin from $B$. gargarizans (GenBank accession No. EU661596) was used as an endogenous control. All qPCR assays were performed using an ABI PRISM ${ }^{\circledR} 7500$ Fast RealTime PCR System (Applied Biosystems, USA) in $20-\mu \mathrm{L}$ reactions. Each $20-\mu \mathrm{L}$ reaction contained $2 \mu \mathrm{L}$ template cDNA, $10 \mu \mathrm{L}$ THUNDERBIRD ${ }^{\circledR}$ SYBR $^{\circledR}$ qPCR Mix (Toyobo, Japan), and $0.5 \mu \mathrm{M}$ each forward and reverse primer. Three replicates were included, and qPCR Ct values were analyzed using the $2^{-\Delta \Delta C t}$ method (Shi et al., 2013) and further tested by performing a one-way analysis of variance (ANOVA).

\section{Anti-microbial assay}

Bacteria - Bacillus subtilis (ATCC6633), E. coli DH5a (ATCC69925), Staphylococcus aureus (ATCC2592), and Pseudomonas aeruginosa (ATCC9027) and fungus - Monilia albican (ATCC10231) and Saccharomyces cerevisiae (ATCC9763) were obtained from the American Type Culture Collection. B. subtilis, S. aureus, and P. aeruginosa were cultured in nutrient broth medium, E. coli DH5 $\alpha$ cells were cultured in Luria-Bertani medium, M. albican were cultured in Martin modified medium, and $S$. cerevisiae was cultured in Sabouraud dextrose and yeast extract medium.

To determine the anti-microbial activity of cathelicidin-Bg, stock cultures of microbes (in $-80^{\circ} \mathrm{C}$ ) were grown in medium for $18 \mathrm{~h}$ at $37^{\circ} \mathrm{C}$, diluted to $\mathrm{OD}_{600}=0.2$, and then added to a 96-well plate at $100 \mu \mathrm{L} /$ well. Each microbe was treated with cathelicidin-Bg at a final concentration of $0,0.1,1,10$, or $100 \mathrm{mg} / \mathrm{mL}$. Absorbance was measured at $630 \mathrm{~nm}$ in a Mustikcan FC scanning multiwell spectrophotometer (Thermo Scientific, USA). Ampicillin (Sigma, USA) at a final concentration of $50 \mathrm{ng} / \mathrm{mL}$ was used as a positive control. The experiments were repeated three times.

\section{Tumor inhibition assay}

Human HEK293 and HepG2 cells were purchased from the typical cell culture collection Committee of the Chinese Academy of Sciences Library (Shanghai, China). Cells were cultured in Dulbecco's modified Eagle medium (Hyclone, USA) supplemented with 10\% fetal calf serum (Hyclone, USA) and antibiotics (Sigma, USA) at $37^{\circ} \mathrm{C}$ under a $5 \% \mathrm{CO}_{2}$ and $95 \%$ air humidified atmosphere in a carbon dioxide cell incubator (Sanyo, Osaka, Japan). The medium was replaced every other day.

Human HEK293 and HepG2 cells were seeded on 96-well plates (Corning, USA) at an initial density of $1.0 \times 10^{4}$ cells/well and incubated at $37^{\circ} \mathrm{C}$ for $24 \mathrm{~h}$. Cells were treated with cathelicidin-Bg solution at $0,0.5,5,50$, or $500 \mathrm{mg} / \mathrm{mL}$, at $37^{\circ} \mathrm{C}$ for $24 \mathrm{~h}$. According to the manufacturer protocol (Sangon, Shanghai, China), methylthiazolyldiphenyl-tetrazolium bromide (MTT), whose reducing capacity is an indicator of cellular activity, was added to each well and incubated at $37^{\circ} \mathrm{C}$ for $4 \mathrm{~h}$. Formazan solution was added to each well to resolve MTT formazan crystals. Absorbance at $490 \mathrm{~nm}$, indicating cellular activity, was determined on a Mustikcan FC scanning multiwell spectrophotometer (Thermo Scientific, USA). The experiments were repeated three times.

Genetics and Molecular Research 15 (3): gmr.15038481 


\section{Data analysis}

Significant differences between treatments were identified using ANOVA in SPSS 17.0 , and significance was set at $\mathrm{P}<0.05$.

\section{RESULTS}

\section{cDNA sequence of Cathelicidin-Bg}

Full-length cathelicidin-Bg cDNA (accession No. KU556732) was 909-bp long and contained a 498-bp open reading frame encoding a 166-amino acid residue protein. A 132-bp 3'-untranslated region (UTR) and a 279-bp 5'-UTR were identified at the 3'- and 5 '- ends, respectively, of the cDNA sequence (Figure 1). The deduced amino acid sequence was then used to search the National Center for Biotechnology Information database, which revealed a high degree of identity with the cathelicidin sequences of amphibians and other vertebrates. A putative signal peptide was predicted at the $\mathrm{N}$-terminal, and a mature peptide "RPCRGRSCSPWLRGAYTLIGRPAKNQNRPKYMWV" was predicted at the C-terminal (Figure 1).

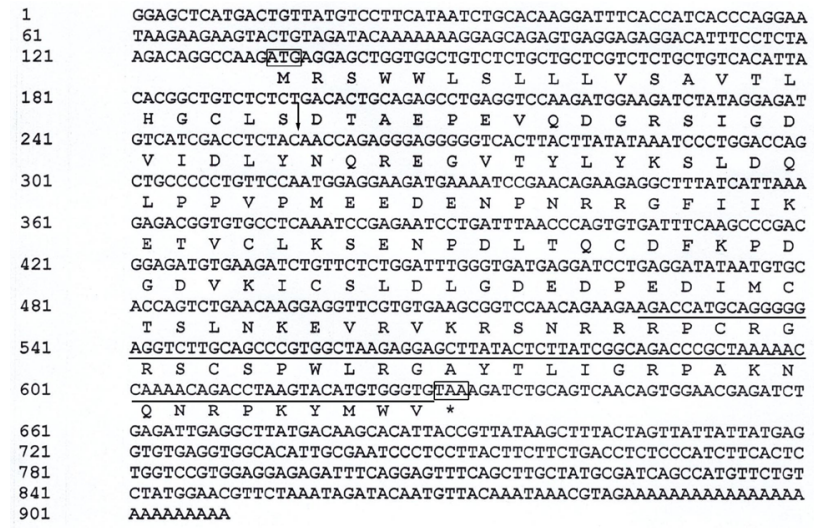

Figure 1. Nucleotide sequence of cathelicidin- $B g$ and its deduced amino acid sequence. Initiation and termination codons are indicated in boxes. The cleavage site of the predicted signal peptide is indicated by an arrow. The mature peptide of the sequence is underlined.

\section{Phylogenetic analysis and multiple alignment}

The EditSeq function in DNASTAR revealed that cathelicidin-Bg was 166-amino acid residues long. The cathelicidin proteins of all selected sequences were classified into two major groups; the cathelicidin-Bg protein clustered with Duttaphrynus melanostictus, Nanorana yunnanensis, Xenopus tropicalis, and Rana catesbeiana, which are all amphibian species (Figure 2A). These results suggest that the results of our analysis were accurate. The amino acid sequence was then compared with those from D. melanostictus, $N$. yunnanensis, $X$. tropicalis, and $R$. catesbeiana by multiple alignments. The results showed that the cathelicidin$\mathrm{Bg}$ amino acid sequence possessed high similarity with those from the other species, suggesting that they have similar functions (Figure 2B).

Genetics and Molecular Research 15 (3): gmr.15038481 

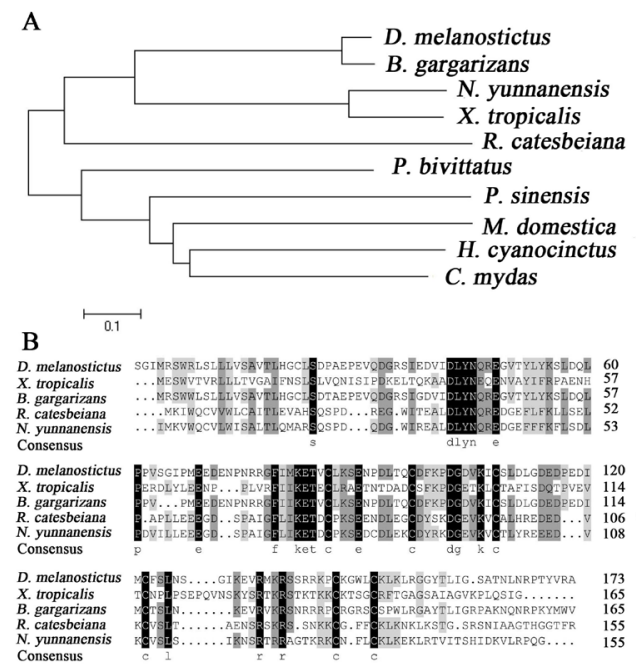

Figure 2. Phylogenetic analysis and multi-alignment of cathelicidin- $B g$ and its homologs from other species. A. Phylogenetic analysis of cathelicidin-Bg and its homologs. All species names are listed on the left of the graph. The following sequences were used in the phylogenetic analysis cathelicidins of Duttaphrynus melanostictus (AJQ20790.1), Bufo gargarizans (KU556732), Nanorana yunnanensis (AFX61592.1), Xenopus tropicalis (XP_004915576.1), Rana catesbeiana (AHW58221.1), Python bivittatus (XP_007443269.1), Pelodiscus sinensis (XP_006129687.1), Monodelphis domestica (XP_003341768.1), Hydrophis cyanocinctus (AKJ54480.1), and Chelonia mydas (XP_007066508.1). B. Multi-alignment of cathelicidin-Bg and its homologs. The amino acid sequences of cathelicidin proteins were obtained from GenBank as follows: D. melanostictus (AJQ20790.1), B. gargarizans (KU556732), N. yunnanensis (AFX61592.1), X. tropicalis (XP_004915576.1), and R. catesbeiana (AHW58221.1).

\section{Expression profile of cathelicidin-Bg}

The expression profile of cathelicidin-Bg in eight cDNA samples was determined by the qPCR method. Our results showed there was dynamic expression of cathelicidin- $B g$ over time from April to November. The expression level of cathelicidin-Bg in April, May, and November was relatively low, while that in August and September was the highest (Figure 3).

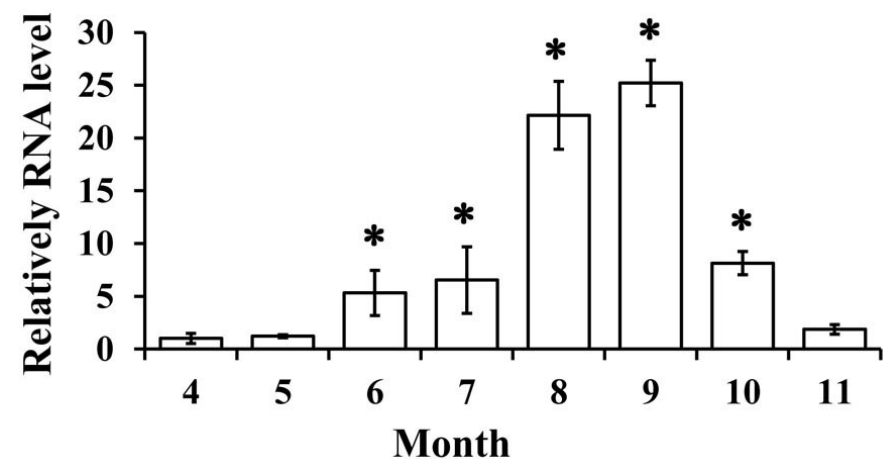

Figure 3. Expression profile of cathelicidin- $\mathrm{Bg}$ over time. Data are reported as means $\pm \mathrm{SE}$ from three independent experiments. $* \mathrm{P}<0.05$.

Genetics and Molecular Research 15 (3): gmr.15038481 


\section{Anti-microbial activity}

Six microbes were used to verify the anti-microbial activity of cathelicidin-Bg. The results showed that cathelicidin-Bg could significantly inhibit the proliferation of two strains of Gram-positive bacteria, B. subtilis and S. aureus, but not that of the other four strains (Figure 4A). The inhibitory effect of cathelicidin-Bg on Gram-positive bacteria has also been shown by the growth curve of $B$. subtilis and $S$. aureus, in which cathelicidin-Bg almost completely inhibited the growth of these two bacterial strains (Figure 4B).

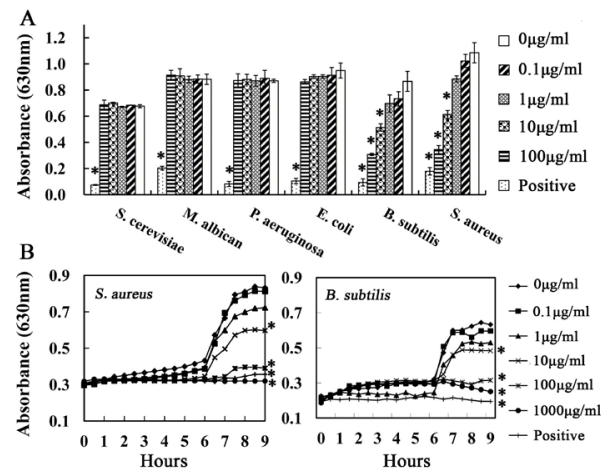

Figure 4. Anti-microbial activity of cathelicidin-Bg. A. Proliferation inhibition of six microbes by cathelicidin-Bg. B. Growth curves of Staphylococcus aureus and Bacillus subtilis treated by cathelicidin-Bg. Data are reported as means $\pm \mathrm{SE}$ from three independent experiments. ${ }^{*} \mathrm{P}<0.05$.

\section{Anti-tumor activity}

The anti-tumor activity of cathelicidin-Bg was verified, since many anti-microbial peptides act as cancer inhibitors. Two kinds of tumor cells were used in this experiment, HEK293 and HepG2 cells. Interestingly, cathelicidin-Bg was able to selectively inhibit the proliferation of tumor cells. The results showed that cathelicidin-Bg could inhibit the proliferation of HEK293, but not HepG2 cells (Figure 5).

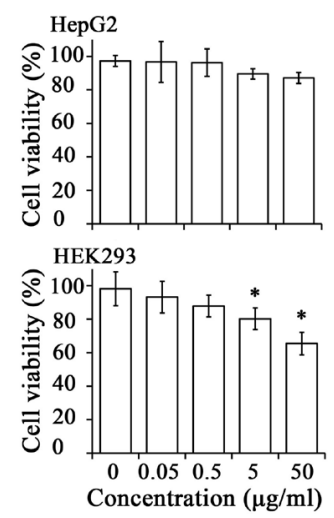

Figure 5. Proliferation inhibition of HEK 293 and HepG2 cells by cathelicidin-Bg. Data are reported as means $\pm \mathrm{SE}$ from three independent experiments. ${ }^{*} \mathrm{P}<0.05$.

Genetics and Molecular Research 15 (3): gmr.15038481 


\section{DISCUSSION}

For a long time, studies on Chan Su have focused on ethanol extracts and many of its components have been identified. However, studies in other Amphibian species have indicated that water extracts, especially for peptides, are the most significant components, which possess anti-microbial and anti-tumor activity. In this study, we identified a gene expressed in the earside gland whose product (a mature peptide) has anti-microbial and anti-tumor activities. The anti-microbial activity differed from that of cathelicidin from Bufo gargarizans (Sun et al., 2015), indicating that AMPs from amphibians possess various functions.

A rapid and effective response to pathogens is essential for the survival of all living organisms. Amphibians live in environments that are full of microbes and have therefore evolved to meet this challenge by producing a large variety of AMPs. AMPs are often secreted from the skin glands of amphibians, such as the serous glands. In the present study, we constructed a cDNA library using total RNA isolated from the ear-side gland of $B$. gargarizans and verified the expression of AMP-encoding genes. Finally, a cDNA, cathelicidin-Bg, was cloned and was found to possess a conserved proregion and a variable $\mathrm{C}$-terminal antimicrobial domain similar to other cathelicidins (Zanetti et al., 1995). In further studies, the mature peptide of cathelicidin-Bg was shown to inhibit the growth of Gram-positive but not Gramnegative bacteria and fungi. In addition, many AMPs showed anti-tumor activity as observed in previous studies (Ohsaki et al., 1992; Lee et al., 2008; Ferreira et al., 2013). In this study, we showed that cathelicidin-Bg could inhibit the proliferation of HEK293 cells but not HepG2 cells; the mechanism(s) involved should be clarified in future studies.

\section{Conflicts of interest}

The authors declare no conflicts of interest.

\section{ACKNOWLEDGMENTS}

Research supported by the Zhejiang Provincial Natural Science Foundation of China (\#LQ14H280007), the Startup Fund (\#2012FR017) from the Zhejiang A\&F University in China, the Startup Fund (\#2012FR080) from the Zhejiang A\&F University in China, and the Student's Science and Technology Innovation Foundation (\#LSY201505) from the Zhejiang A\&F University.

\section{REFERENCES}

Baker MA, Maloy WL, Zasloff M and Jacob LS (1993). Anticancer efficacy of Magainin2 and analogue peptides. Cancer Res. 53: 3052-3057.

Bechinger B, Zasloff M and Opella SJ (1993). Structure and orientation of the antibiotic peptide magainin in membranes by solid-state nuclear magnetic resonance spectroscopy. Protein Sci. 2: 2077-2084. http://dx.doi.org/10.1002/ pro.5560021208

Bevins CL and Zasloff M (1990). Peptides from frog skin. Annu. Rev. Biochem. 59: 395-414. http://dx.doi.org/10.1146/ annurev.bi.59.070190.002143

Chang Y, Zhao Y, Zhan H, Wei X, et al. (2014). Bufalin inhibits the differentiation and proliferation of human osteosarcoma cell line hMG63-derived cancer stem cells. Tumour Biol. 35: 1075-1082. http://dx.doi.org/10.1007/ $\underline{\text { s13277-013-1143-y }}$

Genetics and Molecular Research 15 (3): gmr.15038481 
Chen YY, Lu HF, Hsu SC, Kuo CL, et al. (2015). Bufalin inhibits migration and invasion in human hepatocellular carcinoma SK-Hep1 cells through the inhibitions of NF-kB and matrix metalloproteinase-2/-9-signaling pathways. Environ. Toxicol. 30: 74-82. http://dx.doi.org/10.1002/tox.21896

Ferreira PM, Lima DJ, Debiasi BW, Soares BM, et al. (2013). Antiproliferative activity of Rhinella marina and Rhaebo guttatus venom extracts from Southern Amazon. Toxicon 72: 43-51.http://dx.doi.org/10.1016/j.toxicon.2013.06.009

Giacometti A, Ghiselli R, Cirioni O, Mocchegiani F, et al. (2004). Therapeutic efficacy of the magainin analogue MSI-78 in different intra-abdominal sepsis rat models. J. Antimicrob. Chemother. 54: 654-660. http://dx.doi.org/10.1093/ jac/dkh390

Gibson BW, Tang DZ, Mandrell R, Kelly M, et al. (1991). Bombinin-like peptides with antimicrobial activity from skin secretions of the Asian toad, Bombina orientalis. J. Biol. Chem. 266: 23103-23111.

Hong SH, Kim GY, Chang YC, Moon SK, et al. (2013). Bufalin prevents the migration and invasion of T24 bladder carcinoma cells through the inactivation of matrix metalloproteinases and modulation of tight junctions. Int. J. Oncol. 42: 277-286.

King JD, Leprince J, Vaudry H, Coquet L, et al. (2008). Purification and characterization of antimicrobial peptides from the Caribbean frog, Leptodactylus validus (Anura: Leptodactylidae). Peptides 29: 1287-1292. http://dx.doi. org/10.1016/i.peptides.2008.04.005

Lee HS, Park CB, Kim JM, Jang SA, et al. (2008). Mechanism of anticancer activity of buforin IIb, a histone H2A-derived peptide. Cancer Lett. 271: 47-55. http://dx.doi.org/10.1016/j.canlet.2008.05.041

Lee WH, Li Y, Lai R, Li S, et al. (2005). Variety of antimicrobial peptides in the Bombina maxima toad and evidence of their rapid diversification. Eur. J. Immunol. 35: 1220-1229. http://dx.doi.org/10.1002/eji.200425615

Li J, Ma X, Li F, Wang J, et al. (2010). Preparative separation and purification of bufadienolides from Chinese traditional medicine of ChanSu using high-speed counter-current chromatography. J. Sep. Sci. 33: 1325-1330.

Meng Z, Yang P, Shen Y, Bei W, et al. (2009). Pilot study of huachansu in patients with hepatocellular carcinoma, nonsmall-cell lung cancer, or pancreatic cancer. Cancer 115: 5309-5318. http://dx.doi.org/10.1002/cncr.24602

Miller YE (1990). Bombesin-like peptides: from frog skin to human lung. Am. J. Respir. Cell Mol. Biol. 3: 189-190. http:// dx.doi.org/10.1165/ajrcmb/3.3.189

Ohsaki Y, Gazdar AF, Chen HC and Johnson BE (1992). Antitumor activity of magainin analogues against human lung cancer cell lines. Cancer Res. 52: 3534-3538.

Qiao L, Huang YF, Cao JQ, Zhou YZ, et al. (2008). One new bufadienolide from Chinese drug "Chan'Su”. J. Asian Nat. Prod. Res. 10: 233-237. PubMed http://dx.doi.org/10.1080/10286020701603146

Shi M, Wang YN, Zhu N and Chen XX (2013). Four heat shock protein genes of the endoparasitoid wasp, Cotesia vestalis, and their transcriptional profiles in relation to developmental stages and temperature. PLoS One 8: e59721. http:// dx.doi.org/10.1371/journal.pone.0059721

Shin SY, Kang JH, Jang SY, Kim Y, et al. (2000). Effects of the hinge region of cecropin A(1-8)-magainin 2(1-12), a synthetic antimicrobial peptide, on liposomes, bacterial and tumor cells. Biochim. Biophys. Acta 1463: 209-218. http://dx.doi.org/10.1016/S0005-2736(99)00210-2

Simmaco M, Severini C, De Biase D, Barra D, et al. (1990). Six novel tachykinin- and bombesin-related peptides from the skin of the Australian frog Pseudophryne güntheri. Peptides 11: 299-304. http://dx.doi.org/10.1016/01969781(90)90086-K

Sun T, Zhan B and Gao Y (2015). A novel cathelicidin from Bufo bufo gargarizans Cantor showed specific activity to its habitat bacteria. Gene 571: 172-177. http://dx.doi.org/10.1016/j.gene.2015.06.034

Takeshima K, Chikushi A, Lee KK, Yonehara S, et al. (2003). Translocation of analogues of the antimicrobial peptides magainin and buforin across human cell membranes. J. Biol. Chem. 278: 1310-1315. http://dx.doi.org/10.1074/jbc. M208762200

The State Pharmacopoeia Commission of PR China (2010). Pharmacopoeia of the People's Republic of China (2010 Ed.), Vol. 1. Chemical Industry Publishing House, Beijing, 360-360.

Westerhoff HV, Zasloff M, Rosner JL, Hendler RW, et al. (1995). Functional synergism of the magainins PGLa and magainin-2 in Escherichia coli, tumor cells and liposomes. Eur. J. Biochem. 228: 257-264. http://dx.doi.org/10.1111/ j.1432-1033.1995.00257.x

Zanetti M, Gennaro R and Romeo D (1995). Cathelicidins: a novel protein family with a common proregion and a variable C-terminal antimicrobial domain. FEBS Lett. 374: 1-5. http://dx.doi.org/10.1016/0014-5793(95)01050-O

Zhai XF, Fang FF, Liu Q, Meng YB, et al. (2013). MiR-181a contributes to bufalin-induced apoptosis in PC-3 prostate cancer cells. BMC Complement. Altern. Med. 13: 325. http://dx.doi.org/10.1186/1472-6882-13-325

Zhang DM, Liu JS, Deng LJ, Chen MF, et al. (2013a). Arenobufagin, a natural bufadienolide from toad venom, induces apoptosis and autophagy in human hepatocellular carcinoma cells through inhibition of PI3K/Akt/mTOR pathway. Carcinogenesis 34: 1331-1342. http://dx.doi.org/10.1093/carcin/bgt060

Zhang SJ, Zhang YT, Zhao JH, Shen LN, et al. (2013b). Preparation and in vitro anti-tumor properties of toad venom extract-loaded solid lipid nanoparticles. Pharmazie 68: 653-660.

Zhu Z, Li E, Liu Y, Gao Y, et al. (2012). Bufalin induces the apoptosis of acute promyelocytic leukemia cells via the downregulation of survivin expression. Acta Haematol. 128: 144-150. http://dx.doi.org/10.1159/000339424

Genetics and Molecular Research 15 (3): gmr.15038481 